\title{
Detection and management of alcohol misuse by general practitioners
}

\author{
NP Gurugama, SL Seneviratne, DTS Peiris* and HJ de Silva \\ (Index words: Alcoholism, CAGE and MAST questionnaires, training of general practitioners)
}

\begin{abstract}
Introduction Alcohol misuse and related problems are common in Sri Lanka. The appropriateness of the primary care setting in dealing with alcohol misuse is well recognised, and general practitioners (GPs) constitute an important first contact setting.

Methods One hundred and fifty randomly selected GPs practising in the Colombo and Gampaha districts were given a questionnaire to assess how they detect and manage alcohol misuse, and their attitudes towards persons who misuse alcohol.

Results Seventy per cent of GPs responded [74 male; mean age 42 years (SD 6.7)]. Our results suggest that although a majority (81\%) of GPs were frequently confronted with problems related to alcohol misuse, their efforts to detect the problem and knowledge regarding risk limits of alcohol consumption were poor. Only $25.7 \%$ had even heard of CAGE and MAST questionnaires. The majority of GPs felt inadequately trained to deal with alcohol misuse, but only a few made any self-directed efforts to improve their knowledge and skills regarding its management or referred their patients for specialised care. Participation in preventive programmes was minimal, and many GPs expressed negative attitudes towards persons misusing alcohol.
\end{abstract}

Conclusions The ability of general practitioners to detect and alcohol misuse appears to be inadequate.

\section{Introduction}

Alcohol misuse and related problems are common in Sri Lanka [1,2]. Although no nationwide prevalence studies have been done, biannual spot surveys done by the Alcohol and Drug Information Centre have shown a steady increase in occasional and habitual alcohol use [3]. In a recent survey in a northern suburb of Colombo it was found that prevalence of habitual drinking among males over 15 years of age is $7.3 \%$ (Peiris DTS et al., unpublished data). There has also been a rise in alcohol related hospital admissions and deaths, accompanied by an increase in the allocation from the national health budget for their management [4].

A large number of heavy drinkers are admitted to hospital due to physical complaints, and accidental and non-accidental injuries. It is doubtful whether patients' drinking patterns are explored and identified by the hospital doctors treating them. Alcohol dependence is usually detected only when withdrawal symptoms develop. Such patients are then referred to general hospital psychiatric units. The non-dependent population rarely gets any intervention or feedback about their drinking.

It is well established that early detection coupled with a brief structured intervention given by a person who is able to empathise with problem drinkers can make a significant impact on drinking behaviour and lead to a reduction in alcohol consumption [5]. This kind of brief intervention is relatively simple, takes little time and could be easily administered by any doctor. There is a lack of information regarding this type of intervention targeting common mental illnesses from developing countries [6].

GPs are key persons in the local community and have close contact with patients under their care. They generally know and understand each patient's social and family circumstances. These factors would be invaluable for dealing with people who misuse alcohol. However, to obtain optimal results, GP's must be well motivated to deal with the problem, have a sympathetic attitude towards these persons, and be knowledgeable about how to detect and manage the problem. The aims of this survey were to study the detection and management of alcohol misuse by GPs, and to assess their attitudes towards persons who misuse alcohol.

\section{Methods}

Of the 220 registered GPs practising in the Colombo and Gampaha districts, 150 were randomly selected (using computer generated numbers) and invited to take part in the study. Information was collected using a four-page self-administered questionnaire containing 24 questions. The questionnaire was designed to assess how GPs detect and manage alcohol misuse by their patients. It also included questions regarding attitudes towards persons who misuse alcohol. The questionnaire was pre-tested on a group of 20 GPs registered in the study area, but not included in the present study. Questionnaires were personally handed over to the selected GPs along with a stamped self-addressed envelope to return the completed questionnaire. Two reminders were sent to those who did not return the questionnaires. Completed questionnaires were accepted for up to 4 months after being distributed.

Departments of Medicine and *Psychiatry, Faculty of Medicine, University of Kelaniya, Sri Lanka.

Correspondence: HJ de S, Department of Medicine, Faculty of Medicine, PO Box 6, Ragama, Sri Lanka. Fax: +941958337, e-mail: hjdes@sri.lanka.net (Competing interests: none declared). Received 20 July and accepted 2 August 2003. 
Responses to the questions were entered into a database and analysed using the Microsoft Excel program.

\section{Results}

One hundred and five (70\%) of the 150 GPs [74 males; mean age 42 years (SD 6.7)] responded. The practice size varied from those with less than 10 patients daily to those with up to 150 patients.

Of the GPs who responded, only $16(15 \%)$ routinely inquired about alcohol use from their adult male patients. Most [61 (58\%)] did so only when they strongly suspected that the patient was consuming excessive amounts of alcohol, and $12(11.4 \%)$ if the patient himself indicated that he had a problem with alcohol. None of the GPs routinely asked women patients about alcohol use. The amount of units of alcohol consumed by their patients was routinely calculated by only $11(10.5 \%)$ GPs; $16(15.2 \%)$ did so occasionally. Only $40(38.1 \%)$ GPs could accurately state the recommended low risk levels of alcohol intake for men and women. Twenty seven $(25.7 \%)$ had heard of the alcohol misuse screening questionnaires, CAGE or MAST. Of them, 17 said that they had used the CAGE questionnaire at some time, but only 4 used it on all patients who admitted to drinking alcohol. Sixty (57.1\%) GPs felt that patients usually lied about their drinking.

GPs obtained information regarding alcohol misuse from a variety of sources (Table 1). However, only 11 $(10.5 \%)$ had received some formal training in the subject. Thus, a majority did not feel confident of their ability to detect [63 (60\%)] or manage [58 (55.2\%)] alcohol misuse. When alcohol misuse was detected, $25(23.8 \%)$ GPs stated that they always managed the problem themselves, 20 $(19.1 \%)$ always referred the patient to a physician, 18 (17\%) to a psychiatrist, and $14(13.3 \%)$ to a centre dealing with alcohol related problems. Most GPs felt that training programmes (workshops) on management of alcohol misuse, and more support from social services and organisations that specialise in managing alcohol related problems would benefit both, them and their patients (Table 2).

Table 1. Methods of self-directed learning regarding management of alcohol misuse, Number of GPs $(n=105)$

\begin{tabular}{lcc}
\hline Method & $\begin{array}{c}\text { During } \\
\text { last month }\end{array}$ & $\begin{array}{c}\text { During } \\
\text { last year }\end{array}$ \\
\hline $\begin{array}{l}\text { Attending seminars, workshops, } \\
\text { conferences }\end{array}$ & 16 & 41 \\
Medical journals & 18 & 36 \\
Internet & 14 & 14 \\
TV and radio programmes & 47 & 47 \\
CME programmes conducted by colleges & 2 & 2 \\
During postgraduate studies & 1 & 1 \\
\hline
\end{tabular}

Table 2. Attitudes of GPs towards patients who misuse alcohol and management of alcohol related problems $(n=105)$

Attitudes Agree Uncertain Disagree

Persons who misuse alcohol are rewarding to treat $65 \quad 8$

Persons who misuse alcohol present major management problems

Persons who misuse alcohol are time consuming to deal with 48

I feel confident in my ability to treat persons who misuse alcohol

I feel adequately trained to detect alcohol misuse

More training would encourage me to manage persons who misuse alcohol

Support from local services would make me more willing to deal with persons who misuse alcohol 24

An additional fee would make me more willing to manage persons who misuse alcohol

18

23

64

Responses by GPs to questions regarding their attitudes towards patients who misuse alcohol are given in Table 2. A majority [85 (81\%)] of GPs regularly see individuals with problems related to alcohol misuse, and agreed that a first contact setting was ideal for its detection and for delivering health education. A small proportion [9 $(8.6 \%)]$ of GPs exhibited posters relating to ill effects of misusing alcohol in the patient waiting areas of their clinics, while only $2(1.9 \%)$ had ever got involved in community health education programs regarding the issue.

\section{Discussion}

There is an urgent need to develop strategies for prevention of alcohol misuse in Sri Lanka. Several such strategies have been proposed in the UK [7], and among them is an effort by GPs to carry out a programme of patient education about drinking, to be directed particularly at those who are "at risk" because of their alcohol consumption. The importance of GPs in the detection and care of persons misusing alcohol has been repeatedly emphasised [8,9]. Screening instruments have been developed and tested, and there is a growing body of evidence from several countries favouring the effectiveness of primary care intervention $[7,10]$. The primary care setting is particularly suitable for this work 
because of its easy access to the general population and the educational opportunities offered by consultation with a general practitioner [11]. In addition, patients are often likely to initiate the discussion on the problem in this setting, making behavioural change more likely [12]. For this strategy to succeed GPs must have a good knowledge regarding morbidity from alcohol and a positive attitude towards people misusing alcohol.

Although our GP system is not comparable to the systems in most developed countries, the GP is an important first contact doctor in the system. Ours is the first survey of the management of alcohol misuse by GPs in this country, and to our knowledge, from this part of the world. We especially selected GPs practising in Colombo and Gampaha districts because of the high prevalence of alcohol related problems in these areas $[1,2,4]$. Our results suggest that although GPs were frequently confronted with individuals with problems related to alcohol misuse, their interest in actively detecting the problem and knowledge regarding consumption of alcohol in terms of units and low risk limits were poor. The use of CAGE and MAST questionnaires to screen for alcohol misuse was rare, and although the majority felt that they were not adequately trained to deal with alcohol misuse, only a few referred their patients for specialised care. Like the attitudes expressed by hospital based medical staff in this country [13], many GPs in this study had negative attitudes towards people misusing alcohol. Few made any self-directed efforts to improve their knowledge and skills regarding the management of alcohol related problems. All these factors are likely to result in a poor level of detection and management of alcohol misuse by these GPs.

The majority of GPs stated that access to training programmes and support from social service organisations will encourage them to deal with alcohol misuse more willingly. Providing these would be important steps to improve the current situation, because most GPs recognised the appropriateness of the first contact setting and their own role in dealing with alcohol misuse. As GPs' participation in preventive programmes against alcohol misuse was also minimal, training programmes should focus on this aspect as well.

\section{References}

1. Samarasinghe DS, Dissanayake SA, Wijesinghe CP. Alcoholism in Sri Lanka: an epidemiological survey. British Journal of Addiction 1987; 82: 1149-53.

2. Pathmeswaran A. The Pattern and Problems of Alcohol Use in Gampaha District. MD Thesis, Postgraduate Institute of Medicine, University of Colombo, 1997.

3. Alcohol and Drug Information Centre, Colombo, Sri Lanka, 1995-1999.

4. Annual Health Bulletin, Ministry of Health, Sri Lanka, 1998-2000.

5. Austoker J. Reducing alcohol intake. British Medical Journal 1994; 308: 1549-52.

6. Patel V. The need for treatment evidence for common mental disorders in developing countries. Psychological Medicine 2000; 30: 743-6.

7. Wallace P, Cutler S, Haines A. A randomized controlled trial of general practitioner intervention with excessive alcohol consumption. British Medical Journal 1988; 297: 663-8.

8. Anderson P. Managing alcohol problems in general practice. British Medical Journal 1985; 290: 1873-5.

9. Deehan A, Taylor C, Strang J. The general practitioner, the drug misuser, and the alcohol misuser: major differences in general practitioner activity, therapeutic commitment, and 'shared care' proposals. British Journal of General Practice 1997; 47: 705-9.

10. Babor TF, Ritson B, Hodgson R. Alcohol related problems in primary care setting: a review of early intervention strategies. British Journal of Addiction 1986; 81: 23-46.

11. Fullard E, Fowler G, Gray M. Facilitating prevention in primary care. British Medical Journal 1984; 289: 1585-7.

12. Anderson P, Scott E. The effect of general practitioner's advice to heavy drinking men. British Journal of Addiction 1992; 87: 891-900.

13. de Silva HJ, Kasthuriarachchi N, Senevirathne SL, Senarathne DC, Ellawala NS. Suicides in Sri Lanka: points to ponder. Ceylon Medical Journal 2000; 45: 17-24. 